\title{
EL BANCO MUNDIAL Y LA ENSEÑANZA SUPERIOR EN AMÉRICA LATINA
}

John Saxe-Fernández*

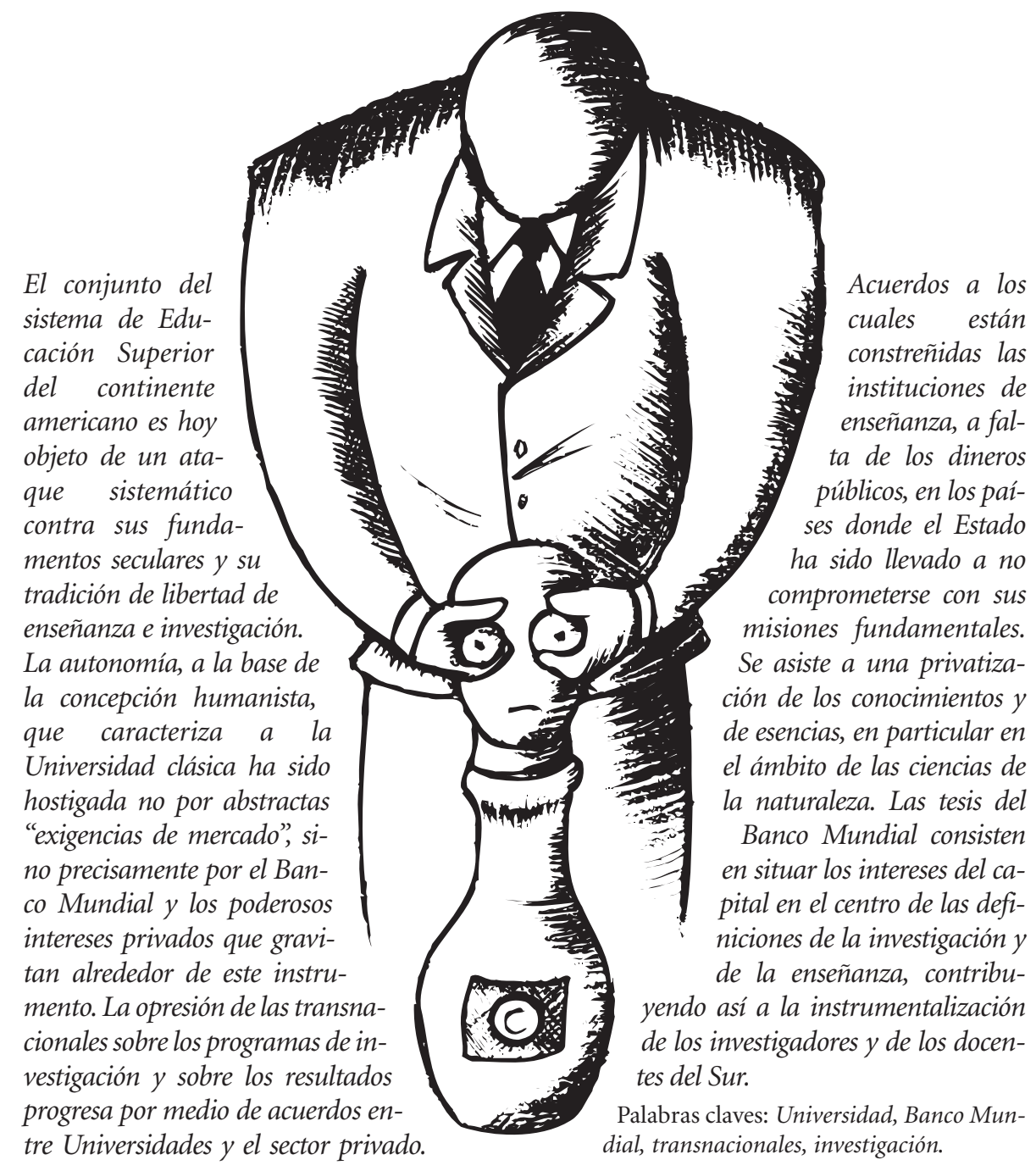

* Profesor e investigador en la Universidad Autónoma de México. 


\section{El fundamentalismo neoliberal}

Uno de los aspectos más apremiantes, a propósito de los problemas y dilemas que encierran toda reflexión sobre la mundialización, el poder y la Educación Superior, es el reconocimiento desde los inicios mismos de este trabajo, de la indispensable apertura a una teorización no sólo económica, sino también política y social, que tiene en cuenta acontecimientos emergentes en un mundo en constante cambio. El punto de vista interdisciplinario da más fuerza a la explicación y permite una mejor comprensión, tanto de la compleja gama de variables implicadas como de su posible evolución en el tiempo. Abordar de esta manera dicho fenómeno es una tarea a la vez, difícil y arriesgada. La Universidad Autónoma de México (UNAM), hoy la institución universitaria de mayor envergadura en América Latina, está sometida, de la misma manera que todo el sistema de Educación Superior de la región, a un ataque sistemático de sus fundamentos seculares y de la tradición de libertad de enseñanza y de investigación. La autonomía, a la base de la concepción humanista de la libertad de enseñanza y de investigación desinteresada que caracterizan la Universidad clásica, está siendo amenazada no tanto por las "exigencias del mercado" o de una abstracta mundialización, sino muy específicamente por el Banco Mundial y los poderosos intereses domésticos e internacionales que se articulan en torno a este centro institucional del poder imperial.

La introducción de los objetivos y de las políticas "de mercado" o, en otras palabras, de esta forma de "fundamentalismo neoliberal" en la investigación y la enseñanza universitarias, representa sin duda alguna un desafío y un escollo a ser vencido en la larga y difícil lucha por la superación de las condiciones alienantes y de opresión que sufre la humanidad. El "modelo estadounidense" contrasta con la experiencia universitaria europea, que ignora virtualmente la existencia de centros privados a este nivel. La inexistencia virtual de Universidades privadas en Europa es un dato de primera importancia, generalmente desconocido por los tecnócratas de los continentes del Sur, que aplican de manera acrítica y mecánica las recomendaciones del Banco Mundial, en particular aquéllas que van en el sentido del desmantelamiento o del debilitamiento de la Universidad pública por medio de la implementación cueste lo que cueste del programa de "Universidades Tecnológicas" en las 
cuatro esquinas del país. Estas últimas están destinadas a formar personal en tareas técnicas inmediatamente requeridas por las empresas, es decir personas que salen de la Universidad con una visión acrítica y estrecha, sin una formación de tradición humanista sobre la historia y los fundamentos del conocimiento científico en general, que debe poseer cualquier profesional de las artes, las ciencias naturales o sociales.

En cuanto creación "universal" y humanista, la Universidad es la antítesis de formulaciones dogmáticas o, si se prefiere un término más cercano a nuestra experiencia, "neo inquisitoriales". Hoy, lo que el director de Le Monde Diplomatique, Ignacio Ramonet, ha caracterizado como "la dictadura del pensamiento único", constituye la principal amenaza para el funcionamiento y la existencia de la Universidad sobre el planeta. Articulado al nivel operacional en torno de las altas esferas gubernamentales, comprometidas hoy con los objetivos y los programas concebidos "en función de las fuerzas del mercado", este fundamentalismo despliega tanto en su retórica como en su práctica lo que los documentos del Banco Mundial consideran como una campaña contra "la Universidad tradicional" y "el poder excesivo de los profesores e investigadores".

\section{La opción del Banco Mundial}

Es en un contexto de estrecha colaboración entre el Estado y el sector privado multinacional, en medio de discursos eufóricos sobre la "mundialización" o las "reformas de mercado", que es preciso proceder para una evaluación seria y objetiva de las intenciones de las empresas transnacionales. Particularmente, aquéllas de los Estados Unidos, que pretenden influenciar, a través de instrumentos como el Banco Mundial, los procesos de toma de decisiones en materia de Educación Superior, sector muy estratégico para el presente y el futuro de nuestros países. En los hechos, el Banco Mundial y el Fondo Monetario Internacional (FMI) funcionan como instrumentos de proyección de la administración estadounidense en América Latina, promoviendo programas de ajuste estructural y de privatizaciones a ultranza de los sectores públicos, incluidos el de la Educación Superior y la Investigación... Todo en nombre de la mano invisible del mercado global.

En nuestras universidades esta "proyección" toma la forma de un esfuerzo sistemático por introducir y colocar los valores, los intereses y las prácticas de la clase empresarial y del mundo de los negocios, por encima 
de los ideales y de los resortes de la Educación Superior que son el interés científico, la investigación fundamental desinteresada y el interés superior de la nación. En un documento titulado "El financiamiento y la administración de la Educación Superior" publicado con motivo de una reunión de la Unesco en París; en 1998, el Banco Mundial explica que la agenda para la reforma educativa "está orientada por el mercado más que por la propiedad pública o por la planificación y la regulación gubernamentales" (Banco Mundial, 1998). Y a continuación se añade que "la dominación casi planetaria del capitalismo $y$ de los principios de la economía neoliberal se encuentra a la base de la orientación por parte del mercado de la educación media-superior y superior".

\section{La finalidad de hacer de la Educación Superior una institución autofinanciable es de hecho despojar a la población latinoamericana de la educación pública, gratuita y obligatoria.}

Los conceptos fundamentales de la agenda del Banco Mundial para la enseñanza y la investigación universitarias son privatización, desreglamentación y orientación por parte del mercado. Sobre el recurrido de la puesta en práctica de esta agenda de reformas, aparece claramente que los problemas, que serán necesarios enfrentar son la universidad tradicional en general y los miembros del personal universitario en particular. Se trata de una verdadera declaración de guerra contra la Universidad y contra el cuerpo de docentes, que tiene por objetivo alejar las funciones universitarias del interés público nacional, poniéndolas al servicio del aparato económico, esencialmente extranjero.

Sin consideración alguna, por la larga experiencia europea, el Banco Mundial impone por medio de préstamos fuertemente condicionados la idea de que la educación y la investigación universitarias son un bien privado -no público- , cuyos problemas pueden resolverse por medio de "soluciones de mercado". Es decir, que su marco es el de una oferta limitada que no está al alcance de todos y que es disponible a cambio de un cierto precio. Además, los consumidores (el mundo de los negocios y de la industria) están "razonablemente bien informados", mientras que los proveedores (administradores y profesores) están "frecuentemente mal informa- 
dos", condiciones ideales para que operen las fuerzas del mercado.

Financiar la demanda significa en la práctica: a) aumentar los derechos de inscripción; b) recubrir el costo total de los estudios; c) poner en práctica medidas de préstamos a los estudiantes; d) percibir los intereses prevalentes sobre el mercado de todos los préstamos; ;) mejorar la cobertura de los préstamos a través de compañías privadas e instaurar un impuesto para los diplomas; f) formar los profesores como jefes de empresa; $g$ ) vender la investigación y los cursos; y h) aumentar el número de instituciones educativas privadas que perciban la totalidad del costo de la enseñanza.

La finalidad de hacer de la Educación Superior una institución autofinanciable es de hecho despojar a la población latinoamericana de la educación pública, gratuita y obligatoria, y así "liberar" la sociedad de esta carga por medio de la privatización de la enseñanza y la investigación. Esto "alivia" las finanzas públicas que, como sabemos, han sido ca- da vez más desviadas hacia gastos improductivos que incrementan el papel tributario de nuestras economías: servicio de la deuda externa, subvenciones a los sectores parasitarios como la especulación bancaria...

Tales son los criterios adoptados por las agencias que promueven y financian la investigación universitaria en América Latina ${ }^{1}$. Ellos constituyen otros tantos mecanismos normativos que influyen de manera significativa, sino determinante, en los programas tanto de las ciencias naturales como de las ciencias sociales, y que reducen, pedazo a pedazo, sector tras sector, la autonomía universitaria ${ }^{2}$. Pero el problema no se limita al hecho que el Banco Mundial debilite la capacidad de los cuerpos universitarios y científicos de América Latina para definir su programa de investigación. Más grave todavía es que una proporción significativa de este programa se aleja de los problemas que afectan nuestras sociedades; por la vía del financiamiento y de la imposición de políticas de mercado, se en-

1 Particularmente el CONICET en Argentina, el CAPES y el CNPQ en Brasil, CONICYT y FONDECYT en Chile o el CONACYT de México.

2 En México el CONACYT, "dependencia” pública a partir de la cual se ejerce el poder presidencial sobre las Universidades mexicanas, se encarga de elaborar los patrones de "excelencia" y los programas doctorales en revistas profesionales. De esta manera se distancia de sus funciones propias, la promoción de la ciencia y de la tecnología, y usurpa responsabilidades que incumben en teoría a los colegios profesorales, representantes de las Universidades públicas del país. Consultar de otra parte los trabajos de Pablo Gentili (2000). 
cuentra confiscado por las fuerzas empresariales que definen los parámetros de acción.

El disfrazamiento lingüístico de este fenómeno es enorme, pues el Banco Mundial lo presenta como el resultado de las exigencias de la mundialización, del mercado global, ante los cuales no habría ninguna otra opción. Como textualmente lo explicitan sus recomendaciones, tal proceso de decisiones "debe ser retirado del Estado y de sus instituciones, $y$ depositado entre las manos de los clientes (estudiantes), de los consumidores (el mundo de los negocios y de la industria) y del público. El Banco Mundial estima que el financiamiento gubernamental de la Educación Superior añadido a la responsabilidad de gobernarla, son en una gran medida responsables de la supervivencia de una educación clásica y elitista que, además es insensible a las reales necesidades de la economía global (desregulada). De ahí la necesidad de reformas presupuestarias" (Banco Mundial, 1998).

El programa neoliberal de las actuales autoridades significa en los hechos el despliegue de una campaña contra la Universidad clásica y lo que el Banco Mundial llama "poder excesivo" del profesorado. Es para ellos indispensable terminar con los criterios tradicionales de evaluación universitaria, con la finalidad de abrir la vía a otros, fundados sobre el "financiamiento al rendimiento". El presupuesto público destinado a la Educación Superior debe estar condicionado a la obtención de resultados comercialmente constatables, esto quiere decir en otros términos que la evaluación estará centrada sobre los criterios y las necesidades determinadas por la clase de jefes de empresa y de los hombres de negocios.

Así, la agenda para la "reforma" (eufemismo para designar un profundo retroceso) quiere poner fin al "presupuesto negociado", por el cual el gobierno financia las instituciones sobre la base de criterios tradicionales, como el número de inscritos y la reputación de la calidad. Según el Banco Mundial se debe abrir la vía al "presupuesto para el rendimiento", de tal manera que "los administradores de las instituciones estén obligados de tomar las decisiones que han sido eludidas hasta ahora, por ejemplo, readecuar los recursos en función de las necesidades de los clientes y de los consumidores" (Banco Mundial, 1998).

La gran batalla del Banco Mundial contra la Universidad -en cuanto concepto aparecido en Europa, en América y en el mundo, como motor del humanismo y de la investigación desinteresada para el bien común, producto de la lucha contra la tiranía 
y el dogma, fundamento de una tradición multisecular centrada sobre la libertad de enseñanza y de investigación- tiende a reducirla al poder evaluador que se atribuye a las "fuerzas del mercado". Así "las instituciones serán obligadas a construir la diferenciación y a terminar con la 'repetición isomórfica de la tradicional Universidad clásica fundada sobre la investigación" (Ibídem, 1998).

He aquí el esquema impulsado por los gobiernos latinoamericanos de orientación neoliberal; es decir, altamente sometidos a la condicionalidad crediticia, gobiernos a su vez avasallados por las neo-oligarquías latinoamericanas (Eduardo E. Saxe-Fernández, 1999) que desmantelan de manera obsesiva los fundamentos materiales de la soberanía nacional; despojando de esta forma a nuestra juventud, además de su propio país, de su porvernir y de su alma.

La misión educadora y los ideales universitarios son colocados bajo las horcas caudinas de las fuerzas del mercado y de lo que llaman mundialización. Hace poco un alto funcionario de la Universidad Autónoma de México ha advertido que se va a proceder a un "recorte" significativo en la investigación. Hecho sin duda deplorado por los tecnócratas del Banco Mundial, que sobre la base de lo que sucede en la Universidad pú- blica en los Estados Unidos saben que la limitación presupuestaria es una manera eficaz de arrastrar al sector hacia temas de investigación con valor comercial, a expensas de la investigación fundamental, de las humanidades y de la enseñanza.

\section{Las implicaciones sobre las funciones de la Universidad}

Las implicaciones de estas políticas son vastas, tanto en términos socioeconómicos y políticos como de civilización. Como lo ha expresado recientemente un especialista en estas cuestiones "la Universidad pública es una institución de Estado, la única consagrada a la compleja acción civilizadora que comprende la formación de profesionales $y$ de intelectuales especializados en la creación, actualización y la expansión de todas las ramas del conocimiento y del arte. Ella está formada en la tradición de los cuerpos de oficios del trabajo intelectual que tienen su origen en la Universitas medieval, y poseen sus propias historias locales en cuanto corporaciones del saber, constituidas en federaciones de corporaciones más limitadas y singularizadas por sus dominios, sus tareas $y$ sus propias jerarquías académicas $y$ burocráticas" (Daniel Cazés, 2000). Thorstein Veblen en 1908, al alba del siglo XX, advertía ya que la incorpo- 
ración de los principios del mercado transformarían la enseñanza y la investigación en "simple mercancía que se produce, evalúa, compra y vende" (Thorstein Veblen, 1965; Arthur J. Vidich, 1994).

Hoy las implicaciones son graves. Se instala la empresa multinacional como el agente que define no sólo lo que se investiga, sino también el cómo se lo investiga y lo que no se debe investigar. Esto se hace a través de los organismos utilizados para promover y financiar la investigación universitaria, de tal suerte que el investigador debe ajustarse a los parámetros establecidos por el aparato de las transnacionales. Se trata de una ingerencia de organismos extranjeros facilitada por la falta de iniciativa, de la que ha dado muestras históricamente la patronal latinoamericana en materia de impulsar la formación de una base nacional de investigación y de desarrollo.

Como bien lo nota Pablo Gentili de la Universidad del Estado de Río de Janeiro, "existe una demanda clara, de parte de ciertas instituciones de Educación Superior, tendiente a ampliar el desarrollo de su programa de investigación científica. Pero ¿cuál es la naturaleza de tales programas en la era neoliberal? En el contexto de la dominación del mundo de los negocios y de las finanzas, la comunidad científica latinoamericana está en camino de perder el poder de definir el programa de investigación de sus propias Universidades... El problema de fondo es este: ¿a quién beneficia? La iniciativa aprivada que se caracteriza por una muy débil inclinación histórica por el progreso científico, está en condiciones de ganar este poder en el contexto de la hegemonía neoliberal hoy sobre América Latina" (Pablo Gentili, 2000:13).

Aunque la adopción de los valores empresariales por parte del sistema universitario en los Estados Unidos no constituye un fenómeno realmente nuevo, el hecho es que actualmente, más que nunca, la Universidad pública se encuentra allí sometida a mayores presiones que minan los objetivos de la enseñanza, y eso al interior de las mismas instituciones. Thorstein Veblen había señalado que la Universidad estadounidense había fracasado en su deber moral, no sólo respecto de sí misma, sino también respecto de la misma civilización que la había concebido y nutrido. C. Wright Mills y después de él otros autores como Seymour Melmen también han puesto en guardia sobre el impacto negativo del sector de la industria militar estadounidense en el financiamiento de la investigación universitaria.

Hoy, cerca del 50\% de toda la investigación que se efectúa en los Es- 
tados Unidos tiene un vínculo directo o indirecto con el aparato de seguridad nacional. Tal y como aparece en las observaciones de C. Wright Mills, la simbiosis entre las grandes empresas privadas y el sector militar es profunda. Recordemos a este propósito la ironía de Marcus Raskin Kennedy: "National Security is Business, Business is National Security" ("La seguridad nacional son los negocios; los negocios son la seguridad nacional'). Tanto en los contratos entre las Universidades y el Departamento de Defensa como en aquellos subscritos con las grandes firmas -farmacéuticas, industriales, etc.- prevalece la norma del secreto; en el primer caso, por razones de seguridad militar, y en el segundo, como una exigencia de la empresa que financia la investigación para preservar todo descubrimiento bajo su estricto control hasta que ella entre en posesión de la patente correspondiente. En los dos casos, es el principio del uso universal y público del conocimiento generado por la investigación científica que ha quedado bajo el fardo.

\section{La influencia de las empresas transnacionales}

La misma experiencia de las universidades de los Estados Unidos da una idea de lo que nos espera y de la deriva hacia la cual nos arrastran las recomendaciones del Banco Mundial. Por ejemplo, en noviembre de 1998 el Colegio de Recursos Naturales de la Universidad de California, en Berkeley, ha subscrito un acuerdo controversial con la firma farmacéutica suiza Novartis. La empresa acuerda un fondo de 25 millones de dólares para financiar las investigaciones del Departamento de Biología. A cambio de estos 25 millones, la Universidad de California concede a Novartis el derecho de negociar las patentes de un tercio de todos los descubrimientos que pueda hacer el departamento mencionado, incluidos aquellos productos de las investigaciones financiadas con fondos del Estado de California o con dineros federales. Además de ello, un gran peso ha sido acordado a la empresa en el aparato de toma de decisiones del departamento, de tal suerte que Novartis influye de manera cuasi determinante sobre la manera como son definidas las prioridades presupuestarias (Eyal Press; Jennifer Washburn, 2000).

La contradicción entre la "Universidad pública" y el hecho de colocar todo un departamento al servicio de una transnacional no ha pasado por alto a los estudiantes, que inmediatamente se reagruparon en el seno de Students for Responsible 
Research. Esta asociación analiza la creciente privatización de factor de la Universidad de California y actúa en coalición con organismos de la Sociedad Civil. Su objetivo es combatir a favor del mantenimiento de una investigación libre de la influencia de los intereses constituidos, o dicho de otra manera, para la defensa de la esencia de la investigación científica. Esto se comprende tanto más si se tiene presente que según los acuerdos entre la Universidad y Novartis una parte significativa de los descubrimientos, pero también los elementos de información que han conducido a ellos, deben permanecer como "secreto industrial" hasta que se conviertan en propiedad privada por medio de las patentes. Recientemente, Novartis ha decidido cambiar de nombre en razón de la mala publicidad que acarreaba este tipo de acuerdos con las universidades estadounidenses.

En el ámbito de la investigación genética, los países capitalistas centrales presionan en la actualidad para introducir mecanismos como los "derechos de autor", los derechos de mejoramiento de plantas, las patentes, las marcas comerciales depositadas, los secretos y procedimientos industriales, todo ello bajo la rúbrica de derechos de propiedad intelectual (TRIPs), con la finalidad de que sean aceptados y subscritos en los tratados por los países con gran biodiversidad como los países latinoamericanos. La cúspide de este edificio ha sido la Convención sobre la Diversidad Biológica que ha entrado en vigor en 1993.

Para conducir este proceso de apropiación y de privatización crecientes de la naturaleza, el Banco Mundial, por medio de la condicionalidad vinculada a todas estas líneas de crédito, favorece la participación de actores privados a la ocurrencia de empresas multinacinales como General Electric, Rockwell Corporation, Monsanto, Bristol Myers, DuPont, General Motors, Hewlett Packard, IBM, Johnson \& Johnson, Merck, Pfizer, Time-Warner, todos miembros del comité de la propiedad intelectual establecido con ocasión de la Uruguay Round y de la entrada en vigor de los TRIPs en 1995.

"En una época en que las ideas son fundamentales para la economía, escriben dos analistas estadounidenses, las Universidades desempeñan inevitablemente una función en el desarrollo. Pero ¿debemos permitir que las fuerzas comerciales determinen la misión y los ideales universitarios? En la Educación Superior hoy las empresas no sólo financian una porción creciente de la investigación, sino que además con frecuencia dictan los términos en los cuales la investigación debe ser conducida... Muchas universidades con presu- 
puestos limitados invierten sus recursos en ámbitos de investigación con orientaciones comerciales, al mismo tiempo que reducen los medios de los departamentos de humanidades y de la enseñanza" (Ibídem, 2000: 41).

El acuerdo establecido entre el Instituto Nacional de la biodiversidad de Costa Rica (INBio) y las empresas farmacéuticas Merck de los Estados Unidos y Sharp \& Dohme de Costa Rica constituyen un caso de estudio del robo de la naturaleza y de su conocimiento. "En este contrato INBio se encarga de entregar miles de pruebas de plantas, animales $y$ de microbios de todo el país, incluidas las zonas protegidas. No importa cualquiera que sea el producto desarrollado a partir de estas pruebas para que pueda ser patentado y comercializado exclusivamente por Merck C. A. a cambio esta compañía ha pagado 1.1 millón de dólares a INBio para la formación de su equipo de investigación. Alrededor de 30 hombres y mujeres costarricenses egresados de las universidades de este país han sido utilizados por INBio como recolectores o paratasonomistas. Se les ha enseñado a recoger muestras en las zonas protegidas y a preceder a preexámenes" (Michel Flitner, 1998: 157).

Lo que hay de grave en este acuerdo INBio / Merck concluido en 1991, es que "ha sido tomado como modelo para montar nuevos contratos en el marco del negocio del robo de la naturaleza" (Michel Flitner, 1998). Es el caso del acuerdo ya mencionado entre Novartis y la Universidad de Berkeley, así como de un gran número de contratos en constante progresión entre diversas empresas multinacionales y las Universidades o institutos de diferentes países.

\section{El presupuesto público destinado a la Educación Superior debe estar condicionado a la obtención de resultados comercialmente constatables}

En México "la contra-reforma agraria de 1992 (modificación del Art. 27 de la Constitución), junto a otros daños a la nación, ha abierto las puertas a los capitales privados para acceder a los recursos bióticos nacionales" (Gian Carlo Delgado Ramos, 2000). De manera idéntica a la experiencia de Novartis con la Universidad de California en un acuerdo que fue establecido en noviembre de 1998, en la Universidad Nacional Autónoma de México, entre el instituto de bio- 
tecnología y la empresa estadounidense Diversa. Ésta, según datos recogidos por Gian C. Delgado, especializada en los organismos vivos en condiciones extremas que promete un gran potencial económico; cuenta ya con más de 700 patentes reconocidas de enzimas (novel enzymes), sin hablar de sus acuerdos con Hoffman-Laroche, Down Chemical Company, Sigma, Celera, entre otras.

Para este analista "lo más grave del acuerdo UNAM/Diversa se encuentra en los contratos paralelos de esta última. Basta mirar la página internet de Celera (empresa estadounidense responsable del desciframiento del genoma humano, que tiene la mayor cantidad de patentes de materiales biológicos, sin mencionar sus múltiples alianzas con Pharmacia -fusión del sector farmacéutico de Monsanto con Upjohn- Novartis, Aventis, entre otros) para encontrar la confirmación de que ésta ha establecido un acuerdo para descifrar diferentes genomas y patentar genes únicos que se encuentran en la 'biblioteca genética' de Diversa. Más alarmante todavía es el acuerdo establecido entre Celera y los Laboratorios Sandía, institución esencialmente militar encargada de algunas de las investigaciones de alta confidencialidad del Pentágono. Es decir, que la UNAM sirve -en términos de ingeniería genética- para proveer muestras biológicas mexicanas con alto potencial comercial a 50 dólares la pieza, 8 según el contrato suscrito), cediendo todo los derechos de patente a Diversa, que, a su vez puede transferirlos a otros, que en el peor de los casos son, ni más ni menos, el departamento de Defensa de los Estados Unidos" (Gian Carlo Delgado Ramos, 2000).

En una investigación en curso, Gian Carlos Delgado indica que, además del acuerdo ya mencionado, existe otro entre el jardín botánico y la Facultad de Química de la UNAM de una parte y el ICBG (International Coopeative Biodiversity Group) de otra parte. Según los informes de este proyecto (ICBG, 1998), este organismo se ha formado en 1992, a partir de la Convención sobre la Diversidad Biológica (CDB) impulsada por el Banco Mundial y apadrinado por los Fondos para el Medio Ambiente Mundial. El grupo está constituido por el Instituto Nacional de la salud de los Estados Unidos (NIH), la Fundación para la ciencia, la USAID (US Agency for International Development), la USDA (US Department of Agriculture), y está coordinado por el Fogarty International Center del NIH. Uno de sus proyectos mundiales sobre las zonas áridas incluye México, Argentina y Chile. En él colaboran la Universidad de Arizona, el G.W. Hansen's Disease Center de Bâ- 
ton Rouge en Louisiana, el Instituto Nacional de Tecnología Agrícola y la Universidad de Patagonia en Argentina, la Universidad Pontificia de Chile, la UNAM, los laboratorios WyethAyers y la American Cynamid Company, ambos estos últimos propiedad de American Home Products.

La contraparte mexicana del proyecto, bajo la dirección del Dr. Robert Bye, miembro del cuerpo administrativo y docente de la UNAM, está tratando de recoger muestras de cactus, xerophitos y otras plantas de los desiertos de Sonora, Sinaloa, Chihuahua, Durango, San Luís Potosí, Puebla, Nuevo León, Oaxaca y Chiapas. Esta contraparte ha almacenado entre 1993 y 1997 alrededor de 3.500 extractos y su contrato se terminó en 2003. El aspecto más peligroso de este acuerdo es el libre acceso de los participantes a los bancos de información genética del jardín botánico de la UNAM, y eso porque según la ICBG misma, el Dr. Bye "está desarrollando las bases de datos más im- portantes de todo el proyecto de las zonas áridas, superando las expectativas que se tenía para los tres países en su conjunto" (ICBG, 1998).

\section{Conclusión}

De esta manera, las tesis de la tecnocracia del Banco Mundial -que nuestros presidentes y Ministerios de Educación, "los country managers del Banco Mundial" han hecho suyos -consisten en colocar los intereses del capital en el centro de las definiciones fundamentales de la investigación y de la enseñanza en nuestros países. Se contribuye de esta suerte a la explotación mundial de la fuerza de trabajo intelectual por medio de la subcontratación, de la cooptación y de la instrumentalización de los investigadores y docentes del Sur. Este proceso acentúa la dependencia neocolonial y defrauda la función social de creación de equidad y de reflexión prospectiva que nuestros pueblos esperan y exigen de la Universidad.

\title{
Bibliografía
}

\author{
BANCO MUNDIAL \\ 1998 Le financement et l'administration de l'éducation supérieur: rapport sur l'état des \\ réformes dans le monde. \\ CAZES, Daniel \\ 2000 "La Destrucción de la Universidad Pública Mexicana", en Fundación Arturo Ro- \\ senblueth, Agravios a la Nación, México, Ed. Galileo.
}




\section{John Saxe- Fernández}

\section{DELGADO RAMOS Gian Carlo}

2000 "La biodiversidad como Materia Prima del Desarrollo de la Biotecnología y la Piratería del Conocimiento Indígena", La Jornada del Campo, n. 85, 10 abril.

FLITNER, Michael

1998 "Biodiversity: of local commons and global commodities", en Michel Goldman (compilador), Pirvatizing Nature, political struggles for the Global Commons, Rutgers University Press, New Bronswick, New Jersey.

GENTILI, Pablo

2000 "Report on the Crisis of Higher Education: The Permanent Crisis of the Public University", Nacla Report on the Americas, vol. XXXIII, n. 4, enero-febrero.

ICBG

1998 Département d'Agriculture de l'Université d'Arizona, htt://ag.arizona.edu/OALS/ICBG/.

PRESS, Eyal \& WASHBURN, Jennifer

$2000 \quad$ "The Kept University", The Atlantic Monthly, vol. 28885, n. 3, marzo.

SAXE-FERNÁNDEZ, Eduardo E.

1999 La Nueva Oligarquía Latinoamericana, San José de Costa Rica, EUNA.

VEBLEN, Thorstein

1965 The Higher Learning in America: A memorandum on the conduct of Universities VIDICH, Arthur J. by businessmen, New York, Augsutus M. Kelley.

1994 "The higher learning in America in Veblen's time and our own", International Jounrnal of Politics, Culture and Society, vol. 7, n. 4, verano. 